Check for updates

Cite this: RSC Adv., 2017, 7, 26468

\title{
Encapsulation and release of Beauveria bassiana from alginate-bentonite nanocomposite
}

Received 21st February 2017 Accepted 12th May 2017

DOI: $10.1039 / c 7 r a 02185 b$

rsc.li/rsc-advances

\author{
D. P. C. Batista, ${ }^{a}$ I. N. de Oliveira, ${ }^{\text {b }}$ A. R. B. Ribeiro, ${ }^{\text {b }}$ E. J. S. Fonseca, ${ }^{\text {b N. S. Santos- }}$ \\ Magalhães, ${ }^{\mathrm{C}}$ J. G. de Sena-Filho, ${ }^{d}$ A. V. Teodoro, ${ }^{d}$ L. A. M. Grillo, ${ }^{\text {a R. S. de Almeida }}{ }^{\mathrm{e}}$ \\ and Camila B. Dornelas (D)*a
}

\begin{abstract}
The present work is devoted to the study of polymer/layered silicate nanocomposite (PLN) of alginatebentonite as a bead matrix for the entomopathogenic fungus Beauveria bassiana strain CPATC032. In particular, we investigate the encapsulation efficiency and surface morphology of beads presenting different concentrations of bentonite, where the effects associated with the drying method of the samples were analyzed. The internal structure of alginate-bentonite beads are studied by means of Xray diffraction and Fourier transform infrared spectroscopy, determining the impact of the drying process on the crystalline structure of bentonite domains inside the polymeric matrix. By using Scanning Electron Microscopy (SEM) and fluorescence microscopy techniques, we identify a monolithic distribution profile for beads inside the PLN matrix, which is independent of the concentration of bentonite and of the drying method employed. However, our results demonstrated that the in vitro release of the fungus is sensitive to the bead preparation method due to the emergence of a barrier phenomenon as the concentration of silicate is increased. Swelling degree and release kinetics were also investigated, where a strong dependence on the drying method was observed.
\end{abstract}

\section{Introduction}

Pest control is an essential task to the modern agriculture due to its relevance for the production efficiency. Indeed, the effective application of pest control practices may be determinant in the success or failure of a specific crop. In this context, pest control practices defined as "ecofriendly" have attracted a remarkable interest in the agricultural settings, being considered a promising approach for the integrated pest management (IPM) programs. $^{1}$

Recently, the use of entomopathogens has emerged as a safe and efficient procedure due to its consolidated application to control insect pests, presenting a minimal or null effect on the non-target species. ${ }^{2}$ For instance, the entomopathogenic fungus Beauveria bassiana infects a wide range of insect pests, including palm weevils Rhynchophorus spp. (Coleoptera: Curculionidae). ${ }^{3}$ Although conidia from $B$. bassiana have been widely used in IPM, this fungus is highly sensitive to abiotic factors. ${ }^{4}$ The main

${ }^{a}$ Departamento de Farmárcia, Universidade Federal de Alagoas, 57072-970, Maceió, AL, Brazil. E-mail: dornelascb@yahoo.com.br

${ }^{b}$ Instituto de Física, Universidade Federal de Alagoas, 57072-970, Maceió, AL, Brazil ${ }^{c}$ Laboratório de Imunopatologia Keizo-Asami, Universidade Federal de Pernambuco, Recife, PE, Brazil

${ }^{d}$ Empresa Brasileira de Pesquisa Agropecuária, Embrapa Tabuleiros Costeiros, 49025040, Aracaju, SE, Brazil

'Instituto de Química e Biotecnologia, Universidade Federal de Alagoas, 57072-970, Maceió, AL, Brazil techniques used to obtain these conidia are based on the multiplication of fungal material in appropriate substrates, particularly in cereals such as rice. More specifically, a conidial suspension is inoculated in the substrate which leads to the growth and the reproduction of the fungus, with the production of a large number of spores. However, several problems have been reported in this procedure due to the absence of a standardization and the lack of information concerning the fungus provenience, quality and effective concentration. ${ }^{5}$ Further, commercial products based on entomopathogens usually present a reduced shelf life, which restrains their market potential and their use by the agricultural community. As a consequence, the development of new preparation strategies that may lead to the improvement of active agents constitutes an important challenge from the economic point of view. ${ }^{6}$

It is well established that the conidia encapsulation in polymeric matrices or in hybrid materials may preserve the intrinsic properties of the fungus, increasing its effectiveness. ${ }^{7}$ One feasible possibility is the encapsulation of microorganisms in biodegradable matrices, such as the alginate.$^{\mathbf{8 9}}$ In particular, the encapsulation of the fungus Rhizopogon nigrescens in beads of alginate preserved the viability of mycelium for eighteen months against twelve months for the non-immobilized fungus under the same storage conditions. ${ }^{10}$ Further, it was observed that the alginate acts as a physical barrier and a humidity controller, which increases the preservation of the fungus. However, it has been shown the need to use other materials to 
overcome some undesired properties of matrices based on alginate ${ }^{8}$ such as low mechanical strength, high porosity and low stability of hydrogel network in the presence of monovalent ions.

Lupo et $a .^{11}$ have demonstrated an effective control of polyphenol release in beads composed of alginate and cocoa extract, considering different gelation methods based on the use of insoluble calcium salts to enhance the structural stiffness. Their results showed that the release of such a compound is governed by the Fick law with a diffusion exponent $n<0.43$, which can be directly related to the presence of calcium in the diffusion medium. In addition to the release of the active agent, physico-chemical properties of the encapsulating matrix can be reasonably adjusted by inserting different materials in the formulation. Using lyophilization techniques, Chan et al. ${ }^{\mathbf{1 2}}$ produced a matrix based on alginate and maize starch to encapsulate Lactobacillus casei, which improved the stability and the viability of the active agent after the storage.

Over the past decade, the modification of polymeric matrices with the addition of clay lamellae has attracted a remarkable interest due to the possibility of developing nanocomposites with improved structural and functional properties. ${ }^{\mathbf{1 3 - 1 5}}$ Although a large variety of clays has been used, a special attention has been devoted to nanocomposites obtained from the introduction of phyllosilicate clays. In particular, this soft material has been denominated as polymer-layered silicate nanocomposite (PLN), because lamellar clays present a stratified structure in a nanoscopic scale, exhibiting self-organization in a two-dimensional form. ${ }^{16}$ A prominent example of phyllosilicate is the bentonite, which consists of a smectic clay presenting mostly montmorillonite (MMT) in its composition. ${ }^{17}$ The use of bentonite in the production of encapsulation matrices based on PLN exhibits several advantages, such as low cost and photo-protection property. ${ }^{18}$ As a consequence, PLNs based on bentonite emerge as an efficient alternative to encapsulate unstable compounds for controlled drug release, like vitamins. Indeed, Kevadiya et al. ${ }^{19}$ leads to demonstrated the successful incorporation of thiamine hydrochloride (vitamin B1) and pyridoxine (vitamin B6) in nanocomposites of alginate and bentonite, obtaining a sustained release of the active agents. Concerning the release of microorganism in polymeric matrix containing bentonite, previous studies reported the release kinetics tends to be slower for microorganisms than for vitamins as the concentration of the bentonite is increase in the matrix. ${ }^{\mathbf{8} 20}$ More specifically, it was observed that the addition of bentonite reduces the permeability of beads due to physical barriers imposed by the lamellae. By varying the relative concentrations of bentonite and alginate, our group previously reported that a barrier phenomenon may affect the release of fungal conidia for high concentrations of bentonite, preserving the fungus stability for several months. ${ }^{21}$

Although the advantages of encapsulation of $B$. bassiana in alginate matrices containing bentonite have been widely studied, the connection between the release mechanism of the fungal conidia and the swelling of the matrix in beads has not been explored yet. The present work is devoted to the characterization of the release mechanism of $B$. bassiana conidia in vitro. In particular, we investigate how the composition and drying method of the beads affect the growth and release of the bioactive agent, which are important features to the efficient application of such an entomopathogenic fungus in the control of insect pests. It is emphasizing the role played by the swelling of the matrix in beads to the hyphal growth. By using different experimental techniques, the morphology of the bead, the hyphal growing, and the release time evolution are studied. Our results demonstrate that a reduction in the hydration capacity of the beads may promote a delay in the release of the fungal conidia.

\section{Materials and methods}

\subsection{Microorganism and culture medium}

The CPATC032 strain of Beauveria bassiana was supplied by Embrapa Tabuleiros Costeiros, Brazil. The B. bassiana conidia present globular shape with diameter in the range of 1.5 to 2 $\mu \mathrm{m} .{ }^{22,23}$ The sample was obtained and isolated from the palm weevil Rhynchophorus palmarum, which is naturally found infected in the Brazilian northeastern. The isolated agent was seeded in Petri dishes containing a nutritive medium of PotatoDextrose-Agar (PDA) (Himedia, India) and kept in a stove (Solab, Brazil) at a temperature of $28( \pm 1){ }^{\circ} \mathrm{C}$, with a photoperiod of 12 hours. In formulation preparation, suspensions obtained from the scraping of colonies with 10-14 days were used. Such a material was inoculated in distilled water containing Tween $80 ®$ (Sigma-Aldrich, Germany) at $0.02 \%$ as the surfactant. The fungal biomass in suspension was filtrated through sterile gauze in order to extract the mycelium, being subsequently sonicated during 10 minutes aiming to homogenize the sample. The fungal concentration in suspension was determined using a Neubauer's chamber (hemacytometer) (New Optik, Brazil), with the aid of an optical microscope (Metrimpax Hungary/PZOLabimex, Poland). ${ }^{24}$ The viability of such a suspension was evaluated by introducing $50 \mu \mathrm{L}$ on delimited circles in Petri dishes, presenting $2.5 \mathrm{~cm}$ in diameter and containing $5 \mathrm{~mL}$ of the PDA culture medium. The aliquots were softly stirred to spread the conidia, avoiding the cluster formation. Such dishes were incubated during 17 hours in order to verify the germination. By counting 200 conidia in four different areas of the same aliquot, we were able to classify them as viable when germinal tube presented a length greater than the conidia diameter. $^{25}$

\subsection{Preparation of formulations}

The PLN formulations were prepared following the procedure described in ref. 19, with the encapsulation of fungus being carried out under sterile conditions. In particular, an aqueous solution of sodium alginate was prepared in a concentration of $1 \mathrm{wt} \%$. Sodium alginate was purchased from Sigma-Aldrich (Germany). Aqueous suspensions of sodic bentonite were prepared in different concentrations and subsequently mixed with alginate solutions. Such a clay was acquired from Bentec Laviosa Chimica Mineraria S.p.A (Italy). Each bentonite-alginate mixture was stirred for 4 hours to obtain a fully blended 
Table 1 Labeling of beads based on their composition. The yield percentage, the encapsulation efficiency, and the size of beads are presented

\begin{tabular}{|c|c|c|c|c|c|c|}
\hline Formulation & Alginate (wt\%) & Bentonite (wt\%) & Yield (wt\%) & Dry method & Size & $\mathrm{EE}\left(\times 10^{8}\right.$ con per $\left.\mathrm{g}\right)$ \\
\hline \multirow[t]{2}{*}{$\mathrm{AB} 0$} & \multirow[t]{2}{*}{1.0} & \multirow[t]{2}{*}{0.0} & \multirow[t]{2}{*}{70.0} & Air flow & 869 & 1.1 \\
\hline & & & & Lyophilization & 1560 & 3.0 \\
\hline $\mathrm{AB} 4$ & 1.0 & 4.0 & 99.7 & Lyophilization & 2112 & 11.0 \\
\hline \multirow[t]{2}{*}{ AB6 } & \multirow[t]{2}{*}{1.0} & \multirow[t]{2}{*}{6.0} & \multirow[t]{2}{*}{99.2} & Air flow & 912 & 15.0 \\
\hline & & & & Lyophilization & 2180 & 12.0 \\
\hline
\end{tabular}

solution. The resultant mixtures were labeled as described in Table 1. A fungal suspension was prepared in a concentration of $10^{8}$ con per $\mathrm{mL}$, which was added to the bentonite-alginate solution in a ratio of $1: 1 \mathrm{v} / \mathrm{v}$. The final solution was stirred for 1 hour. After the preparation of the fungal suspension, the extrusion was performed in a calcium chloride solution (Vetec, Brazil) at $0.25 \mathrm{M}$, which resulted in the ionotropic gelation of the alginate and in the formation of swollen microspheres (beads). ${ }^{8}$ The beads were kept in a calcium chloride solution during 30 minutes for their internal gelation. The beads were separated by simple filtration and subsequently washed with deionized water in order to interrupt the incorporation process of calcium ions. Distinct methods were employed to dry the beads: (1) using laminar flow cabinet (Filterflux, Brazil) ${ }^{25}$ for a period of 12 hours at room temperature; (2) lyophilizing frozen beads in liquid nitrogen for 24 hours by using LD1500 Freeze-Dryer (Terroni). The encapsulation efficiency was determined in a Neubauer's chamber under light microscopic observation after disruption $0.1 \mathrm{M}$ phosphate buffer solution $(\mathrm{pH}=7.4){ }^{26}$ Further, control beads were produced without fungus inside them to evaluate the matrices properties. All experiments were performed in triplicate.

\subsection{Structure characterization}

Aiming at the determination of the internal structure of beads, $\mathrm{X}$-ray diffraction studies were carried out using a Shimadzu XRD-3000 diffractometer, with $\mathrm{Cu} \mathrm{K} \alpha$ radiation source $(\lambda=$ $1.5418 \AA$ ) , operating at $30 \mathrm{kV}$ and $30 \mathrm{~mA}$. The diffraction angle, $2 \theta$, was varied from 3 to 70 degrees. The measurements were performed on powder samples.

In order to investigate the chemical and structural changes related to the drying methods of beads presenting distinct formulations, FTIR spectra were recorded using a Thermoscientific Nicolet iS $10 \AA$, at the wavenumber range region between 4000 and $700 \mathrm{~cm}^{-1}$. All infrared spectra were recorded in the attenuated total reflectance mode at $8 \mathrm{~cm}^{-1}$ intervals and 16 scans.

\subsection{Morphology of the beads and conidia distribution}

After the drying process, the anisotropy in the bead shape was computed as follows:

$$
\text { Sphericity factor }(\mathrm{SF})=\frac{d_{\max }-d_{\min }}{d_{\max }+d_{\min }},
$$

where $d_{\max }$ and $d_{\min }$ are respectively the largest and the smallest orthogonal diameters of the bead. ${ }^{12}$ The beads diameter has been determined based on analysis of the microscopy images of the samples.

The analysis of the morphology of the beads was characterized using a Shimadzu SSX-550 scanning electronic microscope (SEM). Samples were coated with gold using a Sanyu Quick coater SC-701, operating at a current $10 \mathrm{~mA}$ during 8 minutes. The beads were sectioned with a scalpel to enable the visualization of their internal structure.

The fungal conidia in the matrices were doped with propidium iodide (PI) aiming their visualization by using fluorescence microscopy. Propidium iodide is the standard dye used in the visualization of the cell wall, which presents an emission at $638 \mathrm{~nm}$ when excited with a $420 \mathrm{~nm}$ light source. ${ }^{27}$ The doping procedure consisted in mixing the fungal suspension and the aqueous solution of the dye in a ratio of $10: 1 \mathrm{v} / \mathrm{v}$, with the final mixture being incorporated into the PLN matrix before the ionotropic gelation. Identical formulations without the bioactive agent were used as negative control. Free conidia were doped with the propidium iodide through the preparation of the microscope slides by direct smear, being used as positive control. Aliquots of $50 \mu \mathrm{L}$ of the fermented biomass were spread on the surface of clean microscope slides, being fixed by slow evaporation with the aid of a Bunsen burner. The fixed region was then flooded with dye solution during 3 minutes, being washed with deionized water to remove fluorophore excess. Finally, microscope slides were dried in a laminar flow cabinet and the region of interest was sealed using a cover slip. Images of the samples were taken using a Nikon Eclipse 50i Fluorescence Microscope.

\subsection{Physisorption analysis}

The samples were previously treated at $50{ }^{\circ} \mathrm{C}$ under vacuum for 2 hours, and then submitted to nitrogen physisorption at $77 \mathrm{~K}$ in an Autosorb-1-C equipment (Quantachrome Instruments). Specific surface areas were determined through the BrunauerEmmett-Teller (BET) isothermic method. ${ }^{28}$ Pore diameter and specific pore volume were obtained from nitrogen adsorption and desorption stages, using the Barrett-Joyner-Halenda (BJH) algorithm. ${ }^{29}$

\subsection{In vitro release}

Samples with 10 beads of each formulation were randomly spread over the whole extension of the Petri dishes containing 
PDA culture medium, being incubated as was described in previous sections. In experiments using lyophilization as the drying method, the control sample consisted in a conidia suspension. The release and growth of conidia were verified in three ways: (1) naked eyes; (2) binocular stereoscopy; (3) scanning electronic microscopy. Aiming at the analysis of the hyphal growth by using SEM, beads were kept in culture dishes for different time periods to obtain different growth levels. The sample was carefully removed from the culture plate with a platinum loop, being dried under filter paper with laminar flow before the gold coating.

\subsection{Swelling kinetics}

The average weight of the samples with 50 beads of each formulation was determined using an analytical balance. Each sample was dipped in distilled water until the stabilization of the water uptake. In periods of 24 hours, beads were removed from the water, being softly dried with filter paper to withdraw the water from the surface sample. After the water removal, samples were weighed again. The increase of the average weight may be associated with the swelling degree (SD\%) of the sample as follows:

$$
\mathrm{SD} \%=\left(M_{\mathrm{s}}-M_{\mathrm{d}}\right) / M_{\mathrm{d}} \times 100,
$$

where $M_{\mathrm{s}}$ is the weight of swollen beads and $M_{\mathrm{d}}$ is the weight of dry beads. ${ }^{30}$

\subsection{Release kinetics}

The release profile of the conidia of the beads was determined through optical microscopy and through optical density of the samples in different periods of hyphal growth. Beads of AB4 and AB6 formulations were sown in nutritive medium, where they were kept until the release and the development of the fungus. Beads images were acquired using an Odyssey FC Dual-mode imaging system. The images of the beads were subsequently analyzed with the Image Studio ${ }^{\mathrm{TM}}$ software. Optical density data were analyzed using a standard theoretical model for the diffusion process in polymeric systems. ${ }^{31}$ This model considers that release kinetics can be described by a simple exponential relation given by:

$$
M(t) / M_{\infty}=k t^{n}
$$

where $M(t) / M_{\infty}$ is the fractional solute release and $t$ is the release time. $k$ is a constant associated with the characteristics of the polymeric system and the drug, while $n$ is the diffusional exponent governing the release mechanism.

\section{Results and discussion}

\subsection{Formulation preparation}

The nanocomposites used in the beads production were prepared with different ratios of polymer and lamellar silicate. ${ }^{21}$ Here, the yield percentage corresponds to the remaining mass of the compounds in the final product. The results of the yield percentage, the encapsulation efficiency, and the size of beads are presented in Table 1 . The yield percentage becomes larger as the silicate concentration is increased which may be related to the enhancement of the drip solution viscosity, with a reduction in the loss of matrix material. ${ }^{20}$ We also observe that the encapsulation efficiency and the size of beads increase as the concentration of bentonite is enhanced.

\subsection{Structure analysis}

X-ray diffraction (XRD) patterns of AB6 beads dried by distinct methods are presented in Fig. 1. It can be noticed that the XRD pattern of beads is identical to that observed for bentonite powder (inset of Fig. 1), presenting several peaks related to the clay composition. ${ }^{21}$ In particular, we can observe that the drying process does not modify the crystalline structure of micronsized domains of bentonite inside the polymeric matrix, preserving the basal spacing $d_{001}=15.26 \AA$, which corresponds to the peak occurring at $2 \theta=5.8^{\circ}$. We can notice the emergence of characteristic peaks of montmorillonite at $19.8^{\circ}, 29.5^{\circ}, 35.1^{\circ}$, and $62.0^{\circ} .{ }^{32}$ Further, it is possible to observe the occurrence of several peaks associated with the presence of quartz and cristobalite in the sample. ${ }^{32}$ The similarity of XRD patterns of bentonite and AB6 beads reveals that the polymeric chains did not promote an exfoliation or an intercalation over the lamellar structure of bentonite, with the internal structure of the beads being independent of the drying method. ${ }^{33}$

In order to characterize eventual effects of the drying methods in the silicate/polymer interaction, we present the FTIR spectra of AB0 and AB6 beads in Fig. 2. The FTIR spectra of AB0 beads present the typical absorption bands at $3340 \mathrm{~cm}^{-1}$ and $2940 \mathrm{~cm}^{-1}$, which represent the stretching vibration of $-\mathrm{OH}$ and $-\mathrm{CH}_{2}$ groups. The absorption band at $1600 \mathrm{~cm}^{-1}$ is associated with symmetric stretching vibrations of the COO- group. The band centered at $1419 \mathrm{~cm}^{-1}$ and $1297 \mathrm{~cm}^{-1}$ correspond to the vibrations of $-\mathrm{OH}$ and $-\mathrm{CH}$ groups in the pyranose rings, while the peak at $885 \mathrm{~cm}^{-1}$ is related to $\mathrm{Ca}-\mathrm{O}$ vibrations. In Fig. 2a, it can be noticed that the drying method does not affect the IR spectrum of ABO beads, which is in good agreement with

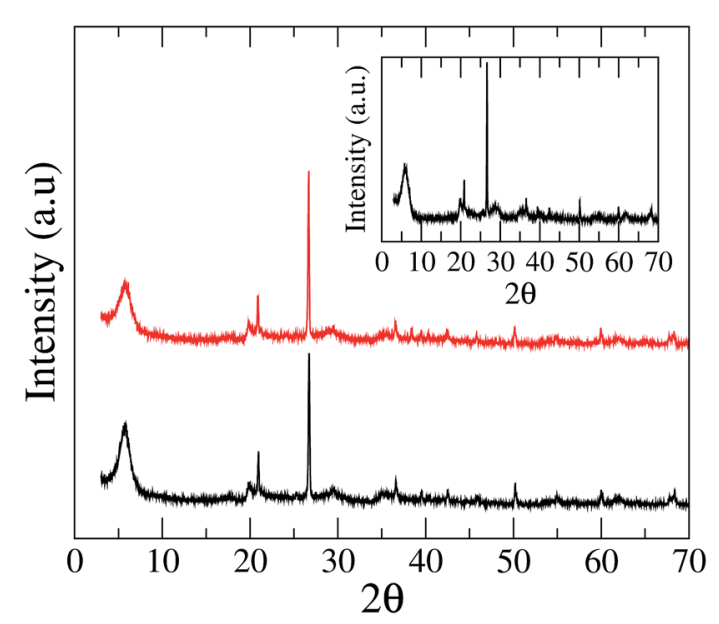

Fig. 1 XRD pattern of AB6 beads dried by distinct methods: air flow (black line) and lyophilization (red line). The inset shows the XRD pattern of bentonite powder in $\mathrm{KBr}$ pellet. 

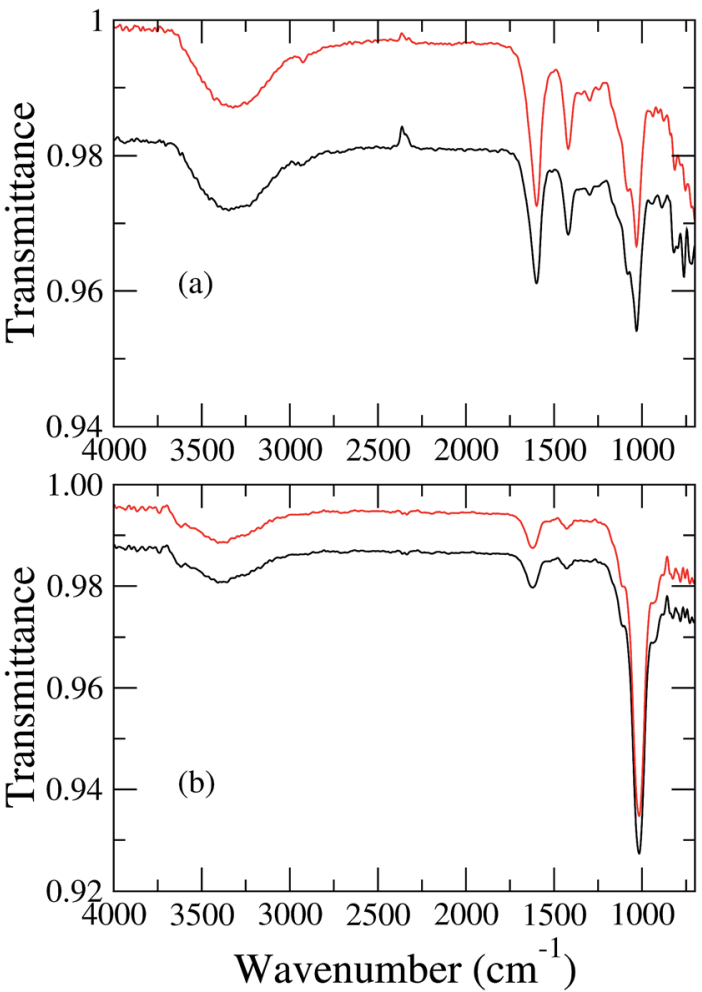

Fig. 2 FTIR spectra of (a) ABO and (b) AB6 beads dried by air flow (black line) and lyophilization (red line) methods.

the previous reports..$^{34}$ In Fig. 2b, we present the FTIR spectra of AB6 beads after air flow and lyophilization drying process. In addition to the absorption bands of the alginate, one can notice the emergence of absorption bands of bentonite. More specifically, the IR spectra exhibit a peak at $3622 \mathrm{~cm}^{-1}$, corresponding to the stretching vibrations of $\mathrm{Al}-\mathrm{OH}$ and $\mathrm{Mg}-\mathrm{OH}$. Further, it is possible to observe absorption bands centered at $1016 \mathrm{~cm}^{-1}$ and $780 \mathrm{~cm}^{-1}$, which are associated with $\mathrm{Si}-\mathrm{O}$ vibrations and OH- bending mode of montmorillonite. ${ }^{19,20}$ Similar to the IR spectrum of $\mathrm{AB} 0$, we can observe that the drying method does not affect the vibrational spectrum of AB6 beads. However, it is important to stress that the addition of bentonite tends to suppress some vibrational modes of the alginates. Indeed, we can notice a pronounced reduction of the peak at $1600 \mathrm{~cm}^{-1}$, which indicates the emergence of an effective interaction between carboxyl groups of alginate with the positively charged sites at the edges of montmorillonite. ${ }^{35}$

\subsection{Morphology of the beads and conidia distribution along the matrix}

In Fig. 3, we present the optical microscopy images of beads presenting different concentrations of bentonite after the drying process by air flow (top panels) and lyophilization (bottom panels). Although an initial spherical shape is observed in all beads after the ionotropic gelation, $\mathrm{AB} 0$ beads exhibit an irregular shape after the drying process, as shown in Fig. 3a and d. As the concentration of bentonite is increased, one can notice that the beads tend to preserve their initial spherical shape after

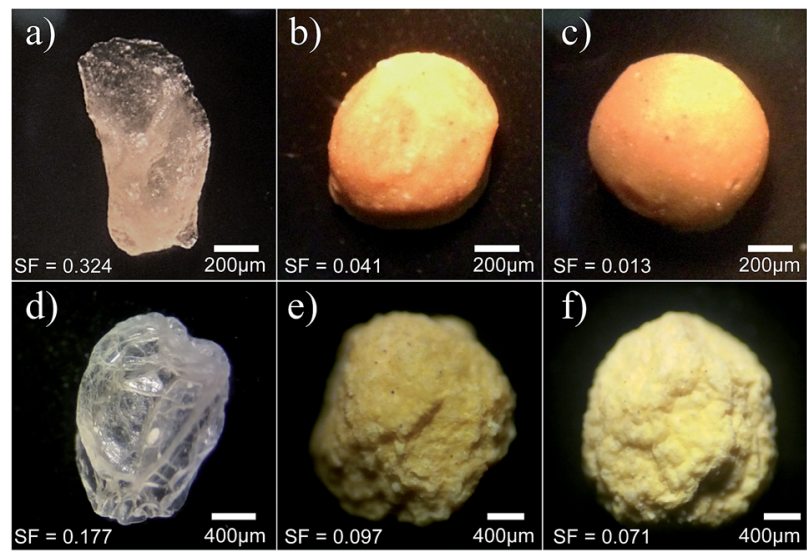

Fig. 3 Optical microscopy images of beads presenting different concentrations of bentonite after drying process by air flow (top panels) and by lyophilization (bottom panels): (a)-(d) ABO, (b)-(e) AB4, and (c)-(f) AB6. In all cases, it is possible to notice that the addition of bentonite tends to preserve the initial spherical shape of the beads after the ionotropic gelation, which is accompanied by an increase of the surface roughness in lyophilized beads.

the drying processes, as exhibited in Fig. 3b, c, e and f. Such composites present a structure where micro-sized bentonite domains are randomly dispersed inside the polymer, ${ }^{21}$ with domains acting as a structural support to it. Indeed, Chan et al. ${ }^{12}$ reported similar results to beads based on alginate polymer, where a factor of sphericity was used to characterize the main differences between beads based on pure alginate and alginate containing starch. In particular, it was observed that the factor of sphericity may vary from null (perfect sphere) in beads with filling materials, to high values in beads containing only alginate. In samples dried by lyophilization, we also notice that the maintenance of the initial spherical shape of the beads after the ionotropic gelation is accompanied by an increase of the surface roughness, as observed in Fig. $3 \mathrm{e}$ and $\mathrm{f}$.

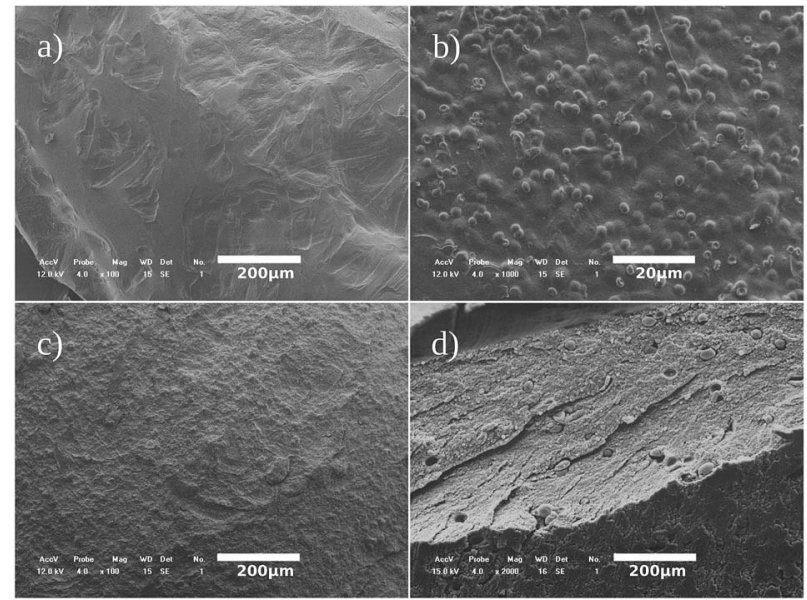

Fig. 4 SEM images of beads presenting different compositions: (a) ABO bead without conidia, (b) AB0 bead with conidia, (c) AB6 bead with conidia, and (d) section of AB6 bead containing conidia. Notice that the bentonite acting as a protective agent for the fungal conidia, preserving their monolithic system of distribution. 
In Fig. 4, we present the scanning electronic microscopy of beads with different composition. For AB0 beads without conidia in their composition, it can be observed that the PLN matrix presents a smooth surface, as shown in Fig. 4a. As the conidia are introduced in the polymeric system, it is possible to notice an enhancement of the roughness at the bead surface due to the uniform distribution of conidia along the matrix, as exhibited in Fig. $4 \mathrm{~b}$. When the bentonite is added in the polymeric matrix, we can no longer identify the conidia on the beads surface, as shown in Fig. 4c. Such an effect reinforces the possibility of using bentonite as a photoprotective agent for the fungal conidia. In order to verify the physical protection of conidia due to the addition of bentonite, we exhibit the SEM images of a transversal section of the beads in Fig. 4d. The PLN matrix presents a monolithic system of distribution, ${ }^{36}$ which is characterized by the uniform distribution of conidia inside the beads. Further, we can notice the existence of vacancies in the PLN matrix due to the extraction of some spores during the bead cut.

In Fig. 5, we present the fluorescence microscope images of conidia doped with propidium iodide dye. We consider the situations of conidia on the surface of a microscope slide and confined them in AB4 beads. These samples were investigated using a UV light as the excitation source. For the bioactive agent on the surface of microscope slides, red bright spots corresponding to the strong emission of PI inside the conidia can be seen, as shown in Fig. 5a. Indeed, we cannot observe the emission of fluorophore inside beads without conidia, as presented in Fig. 5b. This result indicates that an effective interaction takes place between the fluorophore and the bioactive agent. Concerning conidia confined in AB4 beads, we used the PI emission to study the effects associated with the dehydration degree of the samples on the conidia distribution. Despite a few bright spots can be observed, dry and swollen beads exhibit an

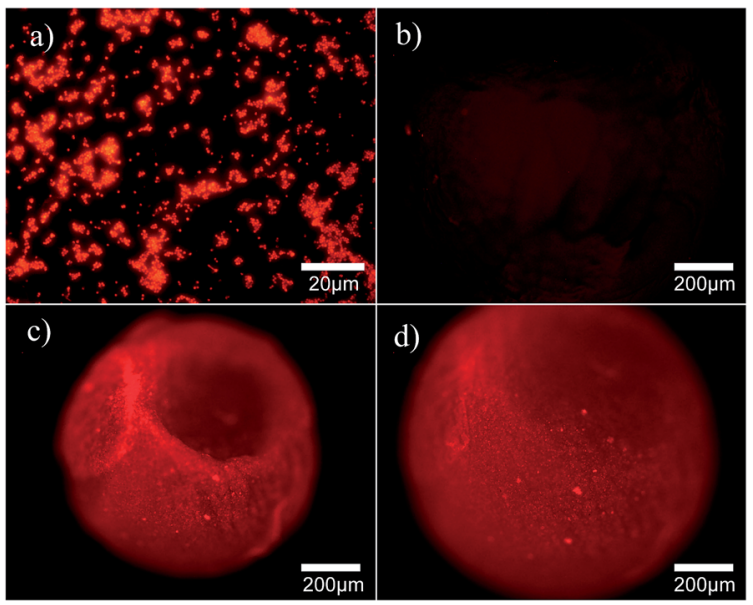

Fig. 5 (a) Fluorescence microscopy image of conidia doped with propidium iodide (PI) dye on the surface of a clean microscope slide. (b) Fluorescence images of bead containing only the solution of PI dye (no conidia). Fluorescence microscopy images of AB4 beads containing conidia: (c) bead dried by air flow and (d) swollen bead. We highlight the conidia distribution inside the beads is preserved during the drying process. almost uniform fluorescence pattern, indicating that conidia doped with PI are regularly distributed inside the beads, as presented in Fig. $5 \mathrm{c}$ and $\mathrm{d}$. Such a result is in agreement with the SEM image of the bead section (see Fig. 4d), showing that the dehydration degree changes the surface morphology of the beads, but it does not modify the spatial distribution of conidia inside them.

\subsection{Physisorption analysis}

The physisorption analysis revealed that the drying method affects the bead structure, with lyophilized beads tending to present a higher surface area than those dried by air flow, as summarized in Table 2 . Such results are directly related to the water sublimation during the lyophilization process, which gives rise to pores and vacancies in the matrix structure, increasing the surface area of beads. ${ }^{12}$ On the other hand, the slow evaporation of water in formulations dried by air flow leads to better accommodation of the matrix structure, resulting in compact beads with a small surface area. Further, we can notice that the increase of bentonite concentration leads to distinct behaviors in samples dried by different methods. In particular, surface area and specific pore volume decreases in samples dried by air flow, while the results for these parameters increase in lyophilized ones. Such trends indicate that the drying method may affect dramatically the release and the swelling phenomena in beads based on polymers and bentonite.

\subsection{In vitro release investigation}

The fermented biomass used in the preparation of the beads containing conidia of $B$. bassiana presented viability rates above $95 \%$, which is essential to the virulence of the bioactive agent. The germination period in suspension of conidia was around 16 hours, being used as the positive control. We have observed that conidia suspensions subjected to lyophilization were not viable, since no germination occurred 72 hours after the sample preparation. The germination of the beads may be observed from the modification of the beads color, which slowly acquire a white coloring until they are covered by the fungal mycelium. In samples dried by air flow, the germination beginning of encapsulated conidia occurred about 24 hours after the seeding process for the formulation without bentonite ( $\mathrm{AB} 0 \mathrm{samples}$ ). As the bentonite concentration is added (AB4 and $\mathrm{AB} 6$

Table 2 BET-specific surface area $\left(S_{\mathrm{BET}}\right)$, specific pore volume $\left(V_{\mathrm{p}}\right)$, pore diameter $\left(D_{\mathrm{p}}\right)$ by nitrogen adsorption of beads with distinct concentrations of bentonite. We consider different drying methods of the beads

\begin{tabular}{lllll}
\hline Formulation & Drying method & $S_{\text {BET }}\left(\mathrm{m}^{2} \mathrm{~g}^{-1}\right)$ & $V_{\mathrm{p}}\left(\mathrm{cm}^{3} \mathrm{~g}^{-1}\right)$ & $D_{\mathrm{p}}(\AA)$ \\
\hline \multirow{2}{*}{ AB0 } & Air flow & 63 & 0.053 & 42 \\
& Lyophilization & 61 & 0.052 & 42 \\
AB4 & Air flow & 41 & 0.035 & 42 \\
& Lyophilization & 62 & 0.070 & 45 \\
AB6 & Air flow & 41 & 0.033 & 42 \\
& Lyophilization & 134 & 0.126 & 43
\end{tabular}


Table 3 Germination period of bead formulations for different drying methods. The germination was identified with naked eyes based on the modification of the beads color, which slowly acquire a white coloring as they are recovered by the fungal mycelium

\begin{tabular}{lll}
\hline Formulation & Drying method & $\begin{array}{l}\text { Germination period } \\
\text { (hours) }\end{array}$ \\
\hline Conidia suspension & Air flow & 16 \\
& Lyophilization & - \\
AB0 & Air flow & 24 \\
& Lyophilization & 50 \\
AB4 & Air flow & 48 \\
& Lyophilization & 48 \\
AB6 & Air flow & 48 \\
& Lyophilization & 24 \\
\hline
\end{tabular}

formulations), we verified a pronounced delay in the germination period, which takes place 48 hours after the seeding process. This result may indicate the emergence of a barrier effect as the concentration of bentonite is enhanced, which is an important feature aiming at release applications.

A distinct scenario has been observed for beads dried by lyophilization method, with the fungus germination becoming faster as the bentonite concentration increases. More specifically, lyophilized AB6 beads presented a germination period around 24 hours, while lyophilized AB0 beads exhibited a germination period close to 50 hours. Table 3 summarizes the germination periods of each sample. It is important to stress that the lyophilization process consists of freezing beads with liquid nitrogen, causing damage to the conidia. The damage caused by the lyophilization process is more pronounced in samples presenting low concentrations of bentonite, due to the reduction of the silicate protection of the beads. Such effect was verified from viability tests in conidia suspensions incorporated in matrices to form beads. This result supports that the silicate may act as a protective material in this case, maintaining the viability of encapsulated conidia against the effects of extreme low temperature in the bioactive samples.

The analysis of the optical images of beads allows us to estimate roughly the beginning of germination process. An accurate determination of the germination beginning can be obtained by using SEM technique, as shown in Fig. 6. Here, we present SEM images of the in vitro release in AB4 beads containing conidia in distinct germination stages. The stereoscopic images of beads are shown in the inset. The growth of conidia leads to significant modifications in the surface morphology of beads, as presented in Fig. 6a-d. SEM images reveal that pronounced changes take place on the bead surface even in the first stages of the germination, while the stereoscopic images of beads seem to be identical, as can be seen in Fig. 6 a and b. In this case, SEM is a sensitive technique to investigate in details the evolution and release of conidia, especially for high concentrations of bentonite. Indeed, SEM technique reduced the time required to identify the hyphal projection in $\mathrm{AB} 4$ and AB6 samples, as shown in Fig. 6. It is important to stress that the growth of the fungal structure of B. bassiana at the bead surface has not been characterized so far. Although stereoscopic

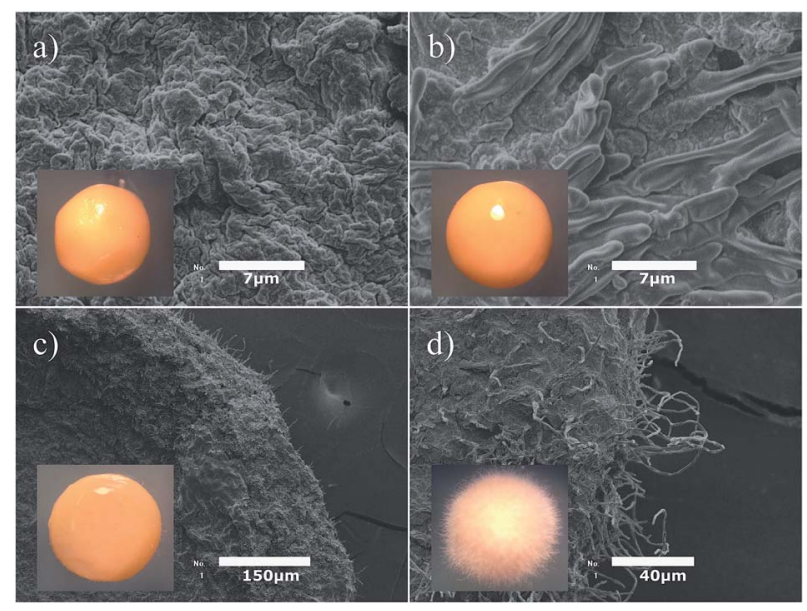

Fig. 6 SEM images of the in vitro liberation of $A B 4$ bead containing conidia in distinct germination stages: (a) 16 hours, (b) 28 hours, (c) 44 hours, and (d) 72 hours after the fungus seeding. The insets show the stereoscopic images of AB4 beads in the same germination stage. It is possible to note that the hyphal growth can be identified 28 hours after the fungus seeding in SEM images, while stereoscopic images seems identical.

microscopy is not sensitive enough to determine the germination beginning with accuracy, such a technique allows us to verify that the swelling process is determinant in the initial development of conidia inside the beads, confirming the relevance of the swelling degree to the release of the active agent. As water is usually a fundamental component for the microorganism growth, the relevance of the water uptake for such products becomes more evident. Indeed, the possibility of controlling the swelling degree from beads formulations may represent an important step to potential application of these composite materials in the delayed release of bioactive agents. ${ }^{37}$

\subsection{Swelling kinetics}

Let us now consider the effects of the bentonite concentration on swelling kinetics of beads dried by air flow and by lyophilization methods. In samples dried by air flow method, we observe that $\mathrm{AB} 0$ beads present a higher swelling degree than formulations containing bentonite, as can be seen in Fig. 7a. Such a result indicates that the polymeric matrix has a high capacity of water uptake..$^{38}$ In fact, the presence of a large number of free carboxylate groups in the alginate polymeric structure contributes to the water uptake. ${ }^{8}$ As the concentration of bentonite is increased, we observed a reduction in the hydration degree of these samples after an initial period of large water uptake. More specifically, the swelling degree decreases from $222 \%$ in $\mathrm{AB} 0$ beads to $178 \%$ in the $\mathrm{AB} 6$ formulation. Concerning the lyophilized samples, we noticed that the introduction of bentonite leads to a huge increase in the water uptake in the first 24 hours. In fact, the water sublimation induces the pores generation in the beads during the lyophilization process. In particular, bentonite reinforces the polymeric structure in the beads, keeping their initial spherical structure which favors the swelling phenomenon. A distinct scenario 

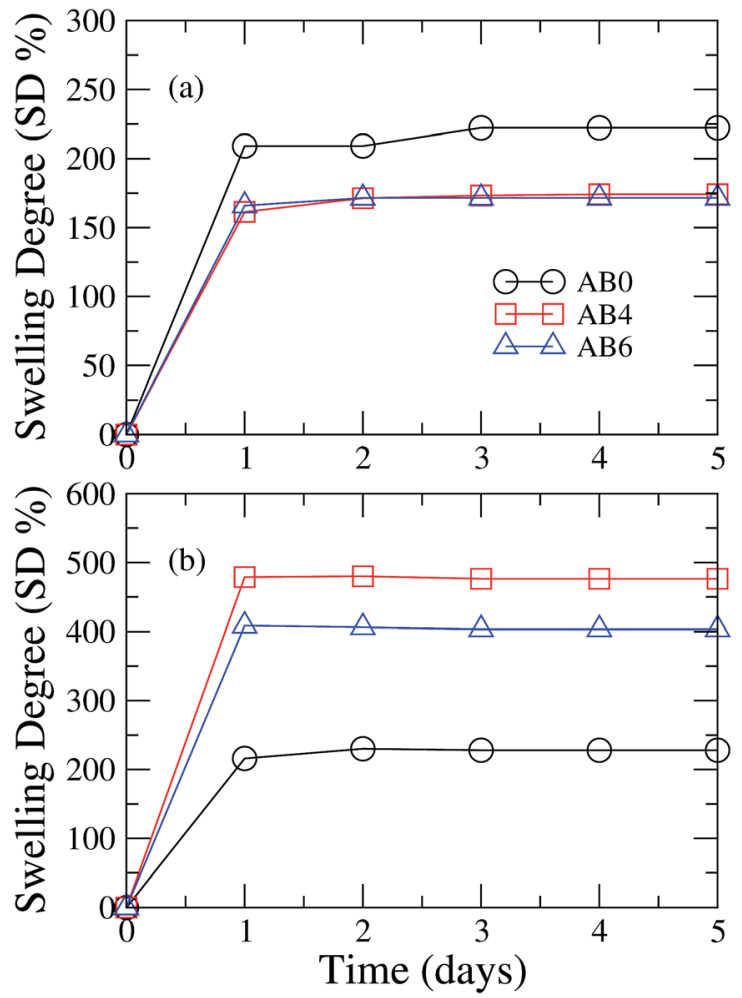

Fig. 7 Swelling degree of beads with different formulations, considering distinct drying methods: (a) air flow and (b) lyophilization. It is possible to note that the beads dried by the lyophilization technique present huge swelling degree.

takes place in $\mathrm{AB} 0$ samples, where the lyophilization process tends to retract the bead structure. More specifically, $\mathrm{AB} 0$ beads do not have enough filler material to maintain their initial spherical shape during the lyophilization, presenting a similar swelling degree to that samples dried by air flow.

The release control of a bioactive agent from the variation of the bentonite concentration in bead formulations was previously reported by $\mathrm{Wu}$ et al. They considered the encapsulation of the Raoultella planticola bacteria inside matrices containing different ratios of alginate, bentonite, and starch. ${ }^{8}$ The reduction of the water uptake was also identified in such formulations, opening the possibility of using these matrices to obtain a system that presents a tunable release. The control of drug release has also been reported by Swamy \& Yun. ${ }^{30}$ They investigated the metformin encapsulation in beads of sodium alginate and carbomethylcellulose, which were obtained through the ionotropic gelation using iron(III) chloride. In particular, a small reduction in the swelling degree was observed as the concentration of carbomethylcellulose in the bead formulation is increased. Similar results were observed as the concentration of gelation agent is enhanced, with the decrease in the swelling degree presenting a noticeable dependence on the $\mathrm{pH} .{ }^{30}$ In all cases, the reduction of the water uptake has been attributed to the enhancement of the polymeric packing associated with the emergence of bonds among adjacent polymeric chains. The swelling degree and the relaxation of the polymeric matrix exert a pronounced influence on the release kinetics of active agents encapsulate in beads. Indeed, the presence of water inside the polymeric matrix promotes the transition from glassy to rubbery states, giving rise to a relaxation of the polymeric network and subsequent release to the active agent. ${ }^{39}$

\subsection{Release kinetics}

In Fig. 8, we present the time evolution of the emitting area of AB4 beads containing $B$. bassiana conidia. Here, we consider the AB4 beads dried by air flow and lyophilization methods. It is important to stress that all measurements were performed after the swelling has been completed, in order to avoid effects associated with the hydration process. In particular, we define the relative emitting area, $\bar{A}(t)$, as

$$
\bar{A}(t)=\frac{A(t)-A\left(t_{\mathrm{SD}}\right)}{A(t \rightarrow \infty)-A\left(t_{\mathrm{SD}}\right)},
$$

where $A(t)$ is the emitting area and $t_{\mathrm{SD}}$ is the time required to complete the swelling process. As one can observe in Fig. 8, the emitting area seems to reach the stationary value several hours after the inoculation time, indicating that the light emission is directly associated with the fungus growth inside the bead. In fact, the fungus development is controlled by the water uptake kinetics, specially in the first 24 hours when the swelling rate is more pronounced. Further, we notice that time evolution of the
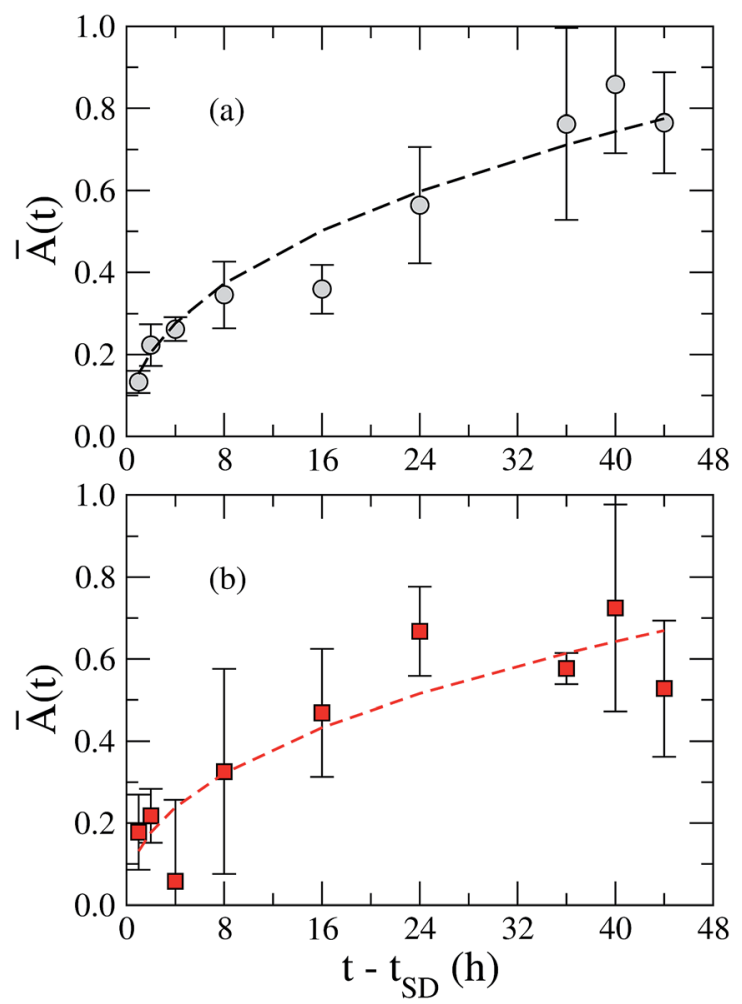

Fig. 8 Time evolution of the relative emitting area, $A(t)$, of $A B 4$ beads dried by (a) air flow (grey circles) and (b) lyophilization (red squares). Dashed lines represent the best fitting curves using the eqn (3), with $n$ $=0.43 \pm 0.04$. Such results demonstrate that the diffusion of the bioactive release mechanism is governed by the Fick transport for AB4 beads, being independent on the drying method. 
emitting area is faster in samples dried by air flow. By considering that the emitting area is proportional to the bioactive agent mass released at time $t$, we can roughly estimate the diffusion exponent $n$. From $\bar{A}(t) \propto t^{n}$, we have estimated respectively $n=0.43 \pm 0.04$ for samples dried by air flow and lyophilization, indicating that the diffusion of the bioactive release mechanism is governed by the Fick transport for AB4 beads. This result demonstrates that the diffusion process of the bioactive agent is not substantially affected by the drying method of the beads.

\section{Conclusions}

In summary, we studied the potential applicability of alginate and bentonite beads for the encapsulation of fungus $B$. bassiana. By using different experimental techniques, we have determined the effects of composition and drying methods of the beads on the growth and release of the bioactive agent. Our results showed that the introduction of bentonite tends to preserve the original surface morphology and the internal structure of the beads during the drying procedure. In particular, we observed an increase in the sphericity of the beads as the concentration of bentonite was enhanced, indicating that the bentonite acts as a structural support to the beads based on the alginate. From electronic scanning and fluorescence micrographs, we noticed a monolithic distribution of the conidia along the beads, with the bentonite acting as a protective agent against thermal damage due to the barrier effect. As a consequence, a pronounced reduction in the germination period was observed in AB6 beads dried by lyophilization, which reflects the huge increase of the water uptake in such samples in comparison to that dried by the air flow method. Indeed, the enhancement of the swelling degree in AB6 samples is mainly associated with the increase of their specific surface area and specific pore volumes as the water sublimation takes place during the lyophilization process. From the analysis of the time evolution of the water uptake and the emitting area of the beads, we have roughly estimated that the release mechanism of the bioactive agent is governed by the Fick transport for beads containing bentonite. This study presented a feasible method for the encapsulation of B. bassiana using alginate/ bentonite composites. Our investigation showed that these matrices provide a microenvironment which is able to protect cells from adverse abiotic factors, increasing the survival of the fungus. Further, our results clarified the relevance of the drying method in the swelling and release properties of beads based on alginate and bentonite. It is important to emphasize that our results were obtained in samples presenting micron-sized domains of crystalline bentonite inside the polymeric matrix. In the case of the delamination of bentonite inside the polymeric matrix, the encapsulation and release of fungus conidia may be strongly affected due to the increase of the diffusion pathways through the nanocomposite. ${ }^{\mathbf{4 0}}$ The possibility of adjusting the release of active principles from these matrices may tailor the development of new strategies to obtain efficient and low cost products aiming at the protection of biological agents used in the control of agricultural pests.

\section{Acknowledgements}

This work was partially supported by Embrapa, CAPES, FACEPE/ CETENE (\# Grant APQ-1456-3.03/15), INCT-FCx CNPq/MCT, and FINEP (Brazilian Research Agencies) as well as FAPEAL (Alagoas State Research Agency).

\section{References}

1 I. Yosha, A. Shani and S. Magdassi, J. Agric. Food Chem., 2006, 56, 8045-8049.

2 S. Toegel, S. Salar-Behzadi, A. Horaczek-Clausen and H. Viernstein, J. Invertebr. Pathol., 2010, 105, 16-23.

3 G. Mazza, V. Francardi, S. Simoni, C. Benvenuti, R. Cervo, J. R. Faleiro, E. Llácer, S. Longo, R. Nannelli, E. Tarasco and P. F. Roversi, Biol. Control, 2014, 77, 83-92.

4 Y. Zhang, J. Zhao, W. Fang, J. Zhang, Z. Luo, M. Zhang, Y. Fan and Y. Pei, Appl. Environ. Microbiol., 2009, 75, 3787-3795.

5 R. Alves, O03 SEB 8th Sincobiol, 2003, vol. 54, p. 42.

6 M. Faria, J. H. Hotchkiss, A. E. Hajek and S. P. Wraight, J. Invertebr. Pathol., 2010, 105, 74-83.

7 S. S. A. Ámora, C. M. L. Bevilaqua, F. M. C. Feijó, M. A. Silva, R. H. M. A. Pereira, S. C. Silva, N. D. Alves, F. A. M. Freire and D. M. Oliveira, Biol. Control, 2009, 50, 329-335.

8 Z. Wu, L. Guo, S. Qin and C. Li, J. Ind. Microbiol. Biotechnol., 2012, 39, 317-327.

9 E. F. Oliveira, H. C. B. Paula and C. M. Paula, Colloids Surf., B, 2014, 113, 146-151.

10 L. P. Oliveira, M. J. Rossi, A. F. Júnior, G. N. S. Filho and V. L. Oliveira, Braz. J. Microbiol., 2006, 37, 251-255.

11 B. Lupo, A. Maestro, J. M. Gutiérrez and C. González, Food Hydrocolloids, 2015, 49, 25-34.

12 E. S. Chan, S. L. Wong, P. P. Lee, J. S. Lee, T. B. Ti, Z. Zhang, D. Poncelete, P. Ravindra, S. H. Phan and Z. H. Yim, Carbohydr. Polym., 2011, 83, 225-232.

13 Y. Ma, F. Shi, Z. Wang, M. Wu, J. Ma and C. Gao, Desalination, 2012, 286, 131-137.

14 S. S. Ray and M. Okamoto, Prog. Polym. Sci., 2013, 28, 15391641.

15 D. R. Paul and L. M. Robeson, Polymer, 2008, 49, 3187-3204.

16 S. S. Ray and M. Bousmina, Prog. Mater. Sci., 2005, 50, 9621079.

17 S. Simon, D. L. Cerf, L. Picton and G. Muller, Colloids Surf., 2002, 203, 77-86.

18 T. Hoang-minh, T. L. Le, J. Kasbohm and R. Gieré, Appl. Clay Sci., 2010, 48, 349-357.

19 B. D. Kevadiya, G. V. Joshi, H. A. Patel, P. G. Ingole, H. M. Mody and H. C. Bajaj, J. Biomater. Appl., 2010, 25, 161-177.

20 Y. He, Z. Wu, L. Tu, Y. Han, G. Zhang and C. Li, Appl. Clay Sci., 2015, 109-110, 68-75.

21 D. P. C. Batista, R. S. Souza, N. S. Santos-Magalhães, J. G. Sena-Filho, A. V. Teodoro, L. A. M. Grillo and C. B. Dornelas, Macromol. Symp., 2014, 344, 14-21.

22 G. Hoog, Stud. Mycol., 1972, 1, 1-41.

23 R. Lohse, D. Jakobs-Schönwandt, S. Vidal and A. V. Patel, Biol. Control, 2015, 88, 26-36. 
24 Ó. Dembilio, E. Quesada-Moraga, C. Santiago-Álvarez and J. A. Jacas, J. Invertebr. Pathol., 2010, 104, 214-221.

25 B. Singh, D. K. Sharma, R. Kumar and A. Gupta, Appl. Clay Sci., 2009, 45, 76-82.

26 N. A. Maftei, A. Y. Ramos-Villarroel, A. L. Nicolau, O. Martínpbelloso and R. Soliva-Fortuny, Food Control, 2014, 41, 27-31.

27 C. M. Rounds, E. Lubeck, P. K. Hepler and L. J. Winship, Plant Physiol., 2011, 57, 175-187.

28 S. Brunauer, P. Emmett and E. Teller, J. Am. Chem. Soc., 1938, 60, 309-319.

29 E. P. Barret, L. G. Joyner and P. P. Halenda, J. Am. Chem. Soc., 1951, 73, 373-380.

30 B. Y. Swamy and Y. Yun, Int. J. Biol. Macromol., 2015, 77, 114119.

31 P. L. Ritger and N. A. Peppas, J. Controlled Release, 1987, 50, 23-36.

32 G. W. Brindley and G. Brown, Crystal Structures of Clay Minerals and Their X-ray Identification, 1980.
33 S. Lonkar, S. Morlat-Therias, N. Caperaa, F. Leroux, J. Gardette and R. Singh, Polymer, 2009, 50, 1505-1515.

34 S. M. Hosseini, H. Hosseini, M. A. Mohammadifar, A. M. Mortazavian, A. Mohammadi, K. Khosravi-Darani, S. Shojaee-Aliabadi, S. Dehghan and R. Khaksar, Int. J. Biol. Macromol., 2013, 62, 582-588.

35 T. Pongjanyaku and S. Puttipipatkhachorn, AAPS PharmSciTech, 2007, 8, E158-E165.

36 B. R. Pezzini, M. A. S. Silva and H. G. Ferraz, Rev. Bras. Cienc. Farm., 2007, 43, 491-502.

37 B. Singh, D. K. Sharma, R. Kumar and A. Gupta, Appl. Clay Sci., 2010, 47, 384-391.

38 S. Rathore, P. M. Desai, C. V. Liew, L. W. Chan and P. W. S. Heng, J. Food Eng., 2013, 116, 369-381.

39 R. H. Juang and D. Storey, J. Controlled Release, 2003, 89, 375-385.

40 P. C. LeBaron, Z. Wang and T. J. Pinnavaia, Appl. Clay Sci., 1999, 15, 11-29. 